ORIGINAL ARTICLE

\title{
Five Years Malaria Trend Analysis in Woreta Health Center, Northwest Ethiopia
}

\author{
Awoke Derbie $^{1 *}$, Megbaru Alemu ${ }^{1}$
}

\footnotetext{
OPEN ACCESS

Citation: Awoke Derbie, Megbaru Alemu. Five Years Malaria Trend Analysis in Woreta Health Center, Northwest Ethiopia. J Health Sci 2017;27(5):465.

doi:http://dx.doi.org/10.4314/ejhs.v27i5.4

Received: March 16, 2017

Accepted: April 22, 2017

Published: September 1, 2017

Copyright: () 2017 Awoke Derbie, et al.

This is an open access article distributed under the terms of the Creative Commons Attribution License, which permits unrestricted use, distribution, and reproduction in any medium, provided the original author and source are credited. Funding: This research was not funded by any grants or another funding agency Competing Interests: The authors declare that this manuscript was approved by all authors in its form and that no competing interest exists.

Affiliation and Correspondence: ${ }^{1}$ Department of Medical Microbiology, Immunology and Parasitology, College of Medicine and Health Sciences, Bahir Dar University, Bahir Dar, Ethiopia "Email: awe.love2000@gmail.com
}

\begin{abstract}
BACKGROUND: An estimated 68\% of the Ethiopian population, living in $75 \%$ of the landmass, is at risk of contracting malaria at any time making it the leading public health problem. The temporal analysis of malaria data could be important to evaluate the performance of malaria prevention programmes. Thus, the aim of this study was to determine the trend of malaria at Woreta Health Center (WHC) over a period of five years.

METHODS: We analyzed the records of 8,057 presumptive malaria patients registered in 2012 to 2016. The following patient data were retrieved from laboratory registration logbook for analysis: sex, age, residence, blood film (BF) microscopy result, type of malaria parasite identified, year and month when the patients visited WHC. Logistic regression was employed to assess the association between potential associated factors and positive $B F$ result; $p<0.05$ was considered significant.

RESULTS: Among the total presumptive individuals, 4447(55.2\%) were females. The prevalence of malaria in each year ranged from $4.1 \%$ to $6.7 \%$. The overall prevalence of malaria was $5.4 \%$ (95\% CI: 4.9\%-5.9\%). The two most important species of malaria parasite identified were $P$. falciparum at $233(53.7 \%)$ and $P$. vivax at 184(42.4\%). Relatively higher proportions of cases were documented in the months of November, December and June (11.1\%, $8.1 \%$ and $7.2 \%$, respectively). Patients who visited the health center in the month of December were $>4$ times more likely to be infected as compared with those who came to the health center in September [AOR: 4.2, 95\%CI (2.374-7.560)]. Females were 1.3 times more likely to be infected than males, [AOR: 1.3, 95\%CI (1.101-1.638)]. Similarly, patients in the age group above 15 were 1.9 times more likely to be infected than individuals $<5$, [AOR: 1.9 95\% CI (1.498-2.455), p value 0.000].

CONCLUSION: In the studied area, malaria remains a major public health challenge. Hence, interventions to decrease the impact of the disease have to be evaluated and strengthened. KEYWORDS: Malaria, trend analysis, Ethiopia
\end{abstract}




\section{INTRODUCTION}

Malaria is one of the major public health problems around the world. Despite being preventable and treatable, malaria continues to have a devastating impact on people's health and livelihoods around the world (1-5). According to the latest available data, about 3.2 billion people, nearly half the world's population, remained at risk of malaria in 97 countries, territories and areas in 2013, and an estimated 198 million cases occurred. In the same year, the disease killed about 584,000 people, mostly children aged under 5 years, in subSaharan Africa (6). In 2015 alone, there were 214 million new cases of the disease and more than 400,000 malaria-related deaths around the globe (5). More than $90 \%$ of clinical cases and death due to malaria occur in Africa (7). Of those Africans who die from malaria each year, most are children under 5 years of age (8) and pregnant women due to low level of immunity (4). In 2015, the African region accounted for approximately 9 in 10 malaria cases and deaths globally (5).

About $75 \%$ of the landmass of Ethiopia is malarious, an estimated $68 \%$ ( $\sim 52$ million) of the population are at risk of contracting malaria $(9,10)$. According to the world malaria report of the year 2016, the number of people living in high transmission ( $>1$ case per 1000 population) and low transmission (0-1 cases per 1000 population) areas in Ethiopia was estimated to be $27,000,000$ $(27 \%)$ and 40,600,000 (41\%), respectively. According to this report, there were 662 reported deaths due to malaria in the country (11). The exact number of people getting sick with and dying of malaria every year in Ethiopia is not well known due to poor documentation. However, it is known that millions of people get sick and tens of thousands die due to malaria every year, and that rates of mortality and morbidity dramatically increase during epidemics $(9,10)$. Plasmodium falciparum and $P$. vivax are the two most dominant malaria parasites in Ethiopia $(12,13)$. They are prevalent in all malaria endemic areas in the country with $P$. falciparum representing about $65-75 \%$ of the total reported malaria cases, relative frequency varying in time and space within given geographical ranges. P. malariae and
P.ovale are rare and account for $<1 \%$ of all confirmed malaria cases. According to the world malaria report of the year 2016, the predominant Plasmodium species identified in Ethiopia were: P. falciparum (64\%) and P.vivax (36\%) (11). The major malaria vector incriminated in Ethiopia is Anopheles arabiensis (12) with A. funestus, A. pharoensis and $A$. nili being secondary vectors (13).

The distribution of malaria in Ethiopia is not uniform (9) and is seasonal in most parts of the country with variable transmission and prevalence patterns affected by the large diversity in altitude, rainfall and population movement. In most parts of Ethiopia, the major transmission periods of malaria occurs from September to December, following the main rainy seasons (JuneSeptember). The minor one is from March to May, following small showers of rain in autumn (12, 14). Since peak malaria transmission often coincides with the planting and harvesting season, and the majority of malaria burden is among older children and working adults in rural agricultural areas, there is a heavy economic burden in Ethiopia (13).

Ethiopia has achieved a remarkable progress in the fight against malaria during the most recent decade through strong preventive and case management interventions with large engagement of the health extension workers (HEWs) and the health development army (HAD) volunteers providing community based care at the household level. The country is also one of the few subSaharan African countries that have shown progress in the fight against malaria (15). Because of the inadequacy of malaria case data from many sub-Saharan African countries including Ethiopia (16), health facility based prevalence studies, like this study, can be used to enhance understanding of the level of malaria and how it is changed over time. The findings would also help policy makers to assess their malaria prevention strategies and their degree of interventions. Moreover, the epidemiological picture of malaria is not yet determined in the study area. Hence, the aim of this study was to determine the trend of malaria at Woreta Health Center (WHC), Northwest Ethiopia, over a period of five years.

DOI: http://dx.doi.org/10.4314/ejhs.v27i5.4 


\section{MATERIALS AND METHODS}

Study design, setting and data collection: In this study, the authors analyzed the records of 8,057 clinically suspected malaria patients who had blood film (BF) examinations for malaria parasites. The study was conducted at WHC. Woreta is a town in Northwestern Ethiopia. According to figures from the Central Statistical Agency of Ethiopia in 2005, the twon had an estimated total population of 26,317 . The town is located approximately $610 \mathrm{~km}$ Northwest of Addis Ababa, the Capital of Ethiopia, having an elevation of about 1828 meters above sea level. The town is wet land with annual temperature range of $18-28^{\circ} \mathrm{C}$.

All patients who visited the health center from 1 September 2011 to 30 August 2016 and had the following registered data were included for analysis: sex, age, residence, $\mathrm{BF}$ microscopy result $(+,-)$, type of malaria parasite identified $(P$. falciparum, $P$. vivax or mixed), year and month when the patients visited the health center. Patients were suspected for malaria when they presented with fever and related clinical symptoms. Data were retrieved directly from laboratory registration logbook using prepared data extraction sheet.

Blood film microscopy procedure: Microscopic examination remains the "gold standard" method for laboratory confirmation of malaria in developing nations, like Ethiopia. The Woreta Health Center is using BF as a standard procedure to diagnose malaria among suspected patients. Capillary blood samples were collected from each presumptive patient for microscopic examination. The finger tip or heal of the child patient was cleaned with swab moistened with $70 \%$ v/v alcohol. After air-drying, a sterile lancet was used to prick the finger or heel, and then squeezed gently to obtain the blood (17). Two blood samples for thick and thin films were prepared on clean slides from each participant according to the standard WHO approved protocol. Slides were labeled properly and air-dried horizontally on a slide tray. Thin films were fixed with absolute methanol immediately after drying, and both thin and thick blood films were stained with $3 \%$ Giemsa solution for 30 minutes. During examination, blood slides were read as either negative, $P$. falciparum positive, P.vivax positive, or mixed infection. Two hundred fields (the equivalent of $0.5 \mu \mathrm{l}$ of thick blood film) were examined at a magnification of $1000 x$ before identifying a slide as negative. If positive, the thin film was read to determine the species $(17,18)$.

Data analysis: All data were entered, cleaned and analyzed using Statistical Package for Social Science (SPSS) software (IBM Corp. Released 2011. IBM SPSS Statistics for Windows, Version 23.0. Armonk, NY: IBM Corp.). Descriptive statistics, like proportion and mean, was used to present data. Logistic regression was employed to assess the association between potential predictors and malaria infection. $P$ value less than 0.05 was considered to indicate statistically significant difference.

Ethical approval and consent: Permission and consent from WHC administrators was obtained to use the data for the research purpose. No patient details that may link to the patient identity, like names, was used and confidentially was maintained.

\section{RESULTS}

Demographic characteristics of study participants: The authors assessed BF microscopic results of 8,057 malaria suspected patients from laboratory reporting logbook processed over a 5 years' period in WHC. Of the total patients registered, 4447(55.2\%) were females. The median age of the patients was 25 years, ranging from 1 month to 85 years. Among the participants, $5062(62.8 \%)$ and $6176(76.7 \%)$ were from rural settings and in the age group of above 15 years, respectively (Table 1 ).

Prevalence of malaria and its trend: The number of clinically suspected malaria patients who visited the WHC over the five years' period was comparable, ranging from 1473(17.5\%) in 2012 to $1766(21.9 \%)$ in 2015 (Figure 1). 
Number of presumptive malaria cases

Number of confirmed malaria cases

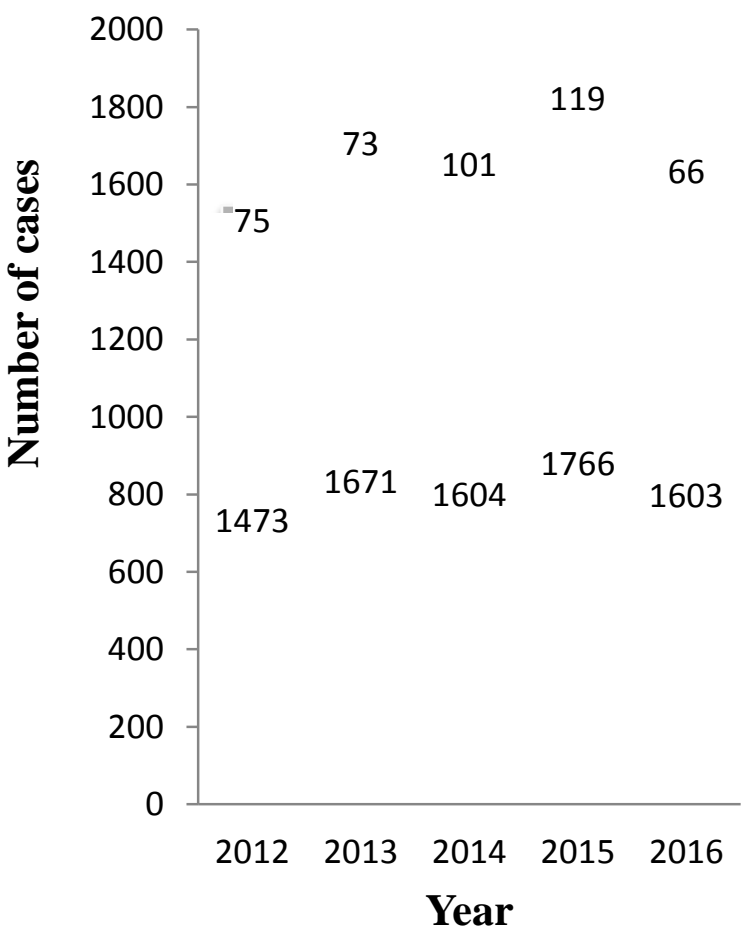

Figure 1: Number of BF confirmed and presumptive malaria cases over the five years period at WHC, 2012-2016

Similarly, the prevalence of BF confirmed malaria cases in each year ranged from $4.1 \%$ in 2016 to $6.3 \%$ in 2014 (Figure 2). The overall prevalence of malaria among suspected cases was $5.4 \%$ (434/8057, 95\%CI: 4.9\%-5.9\%) over the five years' period. When we look at the annual distribution of malaria in the five years' period, relatively higher proportions of confirmed cases were documented in the months of November, December and June at $11.1 \%, 8.1 \%$ and $7.2 \%$, respectively (Figure 3 ). The two most important species of malaria identified were $P$. falciparum, 233(53.7\%, 95\%CI: 49\%-58.3\%), and P. vivax, 184(42.4\%, 95\%CI: $37.8 \%-47.1 \%)$. There were 17(3.9\%, 95\%CI: $2.5 \%-6.6 \%)$ reports of mixed infection with both species (Table 1).
Table 1: Socio-demographic characteristics of the study participants and distribution of malaria at WHC, 2012-2016.

\begin{tabular}{ll}
\hline Variables & $\mathbf{n}(\boldsymbol{\%})$ \\
\hline Sex & \\
Male & $3610(44.8)$ \\
Female & $4447(55.2)$ \\
Total & $\mathbf{8 0 5 7}(\mathbf{1 0 0})$ \\
Age category & \\
$<5$ & $822(10.2)$ \\
$5-15$ & $1059(13.1)$ \\
$>15$ & $6176(76.7)$
\end{tabular}

Mean age 26.1 years

SD 15.4 years

Median age 25.0 years

Range:1month to 85 years

Residence

Rural

Urban

BF result

Positive

Negative

Identified malaria parasites

$P$. falciparum

P. vivax

Mixed

$5062(62.8)$

$2995(37.2)$

$434(5.4)$

7623(94.6)

$233(53.7)$

$184(42.4)$

17 (3.9)

Month

January

$605(7.5)$

Feb

$585(7.3)$

Mar

$467(5.8)$

Apr

$552(6.9)$

May

$668(8.3)$

Jun

$846(10.5)$

Jul

$504(6.3)$

Aug

$492(6.1)$

Sep

$885(11.0)$

Oct

$995(12.3)$

Nov

$660(8.2)$

Dec

$798(9.9)$

Year

2012

$1473(17.5)$

2013

$1671(20.7)$

2014

$1604(19.9)$

$1766(21.9)$

2015

1603 (19.9)

DOI: http://dx.doi.org/10.4314/ejhs.v27i5.4 


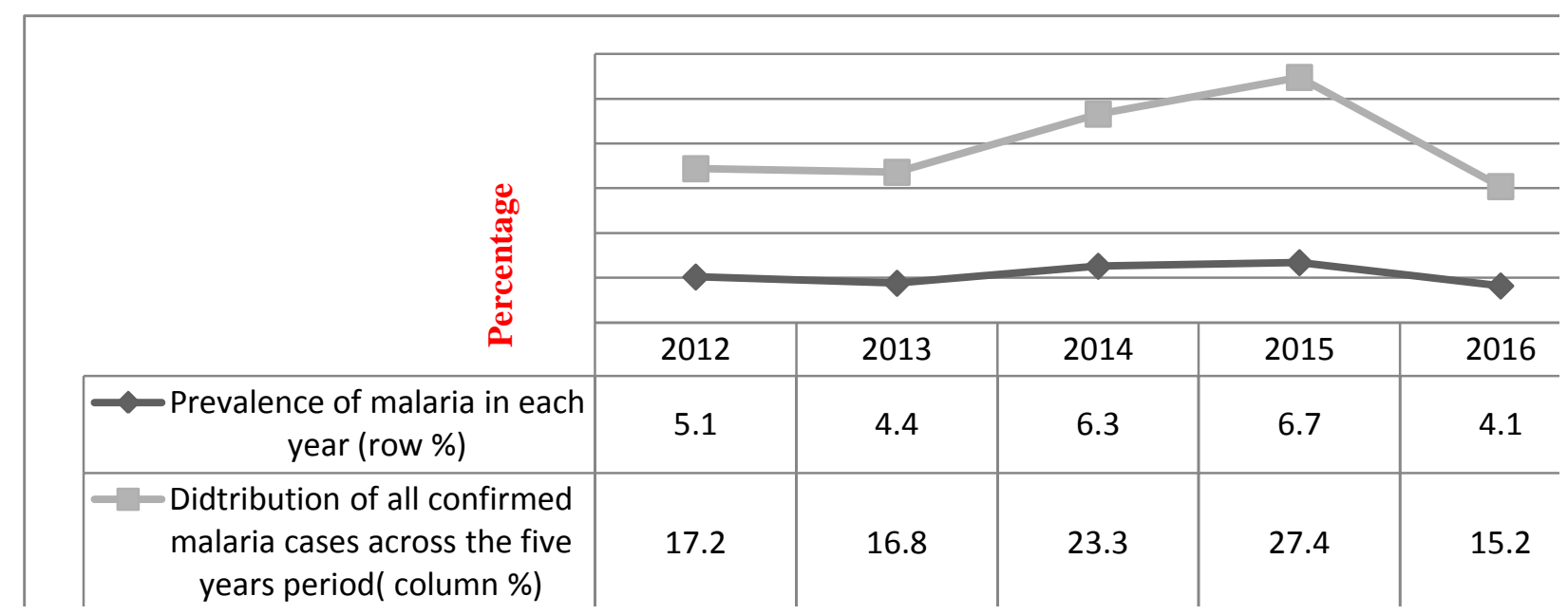

Figure 2: Prevalence of malaria over the five years period at WHC, 2012-2016.

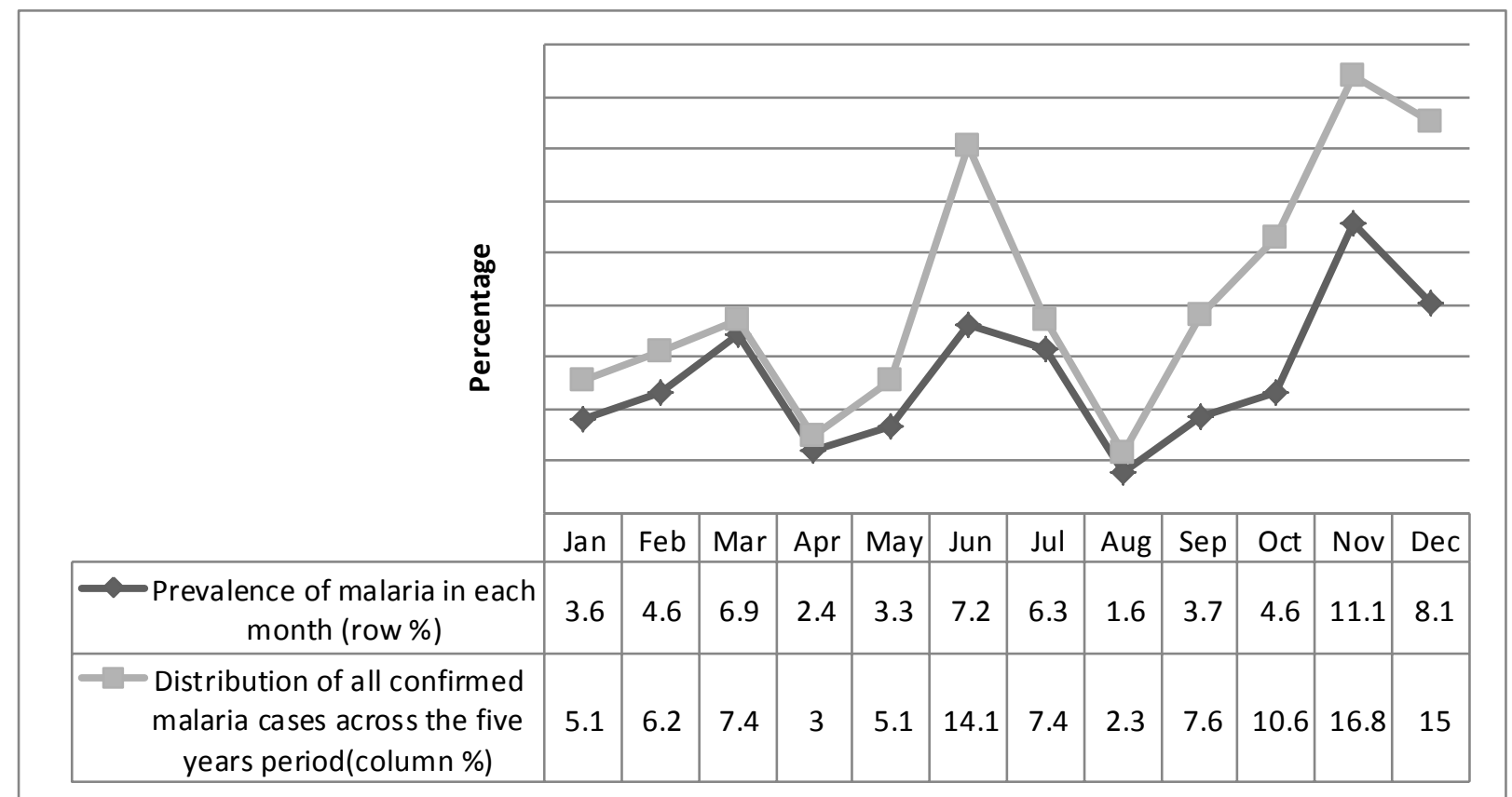

Figure 3: Annual distribution of malaria over the five years period at WHC, 2012-2016.

Factors associated with BF confirmed malaria: In the logistic regression model, it was found that females were 1.3 times more likely to be infected with malaria than males, [AOR: 1.3, 95\% CI (1.101-1.638), $p$ value 0.004]. Similarly, patients in the age group above of 15 were 1.9 times more likely to be positive for malaria than individuals under the age of 5, [AOR: 1.9 95\% CI (1.498-
2.455), $p$ value 0.000]. Moreover, patients who visited the health center in 2016 were 1.6 times more likely to be infected than those who came in 2012 to the center, [AOR: 1.6, 95\%CI (1.1332.214), $p$ value 0.007]. On top of this, patients who visited the WHC in the month of December were more than 4 times more likely to be infected as compared with those who came in September

DOI: http://dx.doi.org/10.4314/ejhs.v27i5.4 
[AOR: 4.2, 95\%CI (2.374-7.560), $p$ value 0.000]. The distribution of malaria also showed a significant difference in the months of November,

Table 2: Logistic regression analysis of factors associated with BF confirmed malaria cases among suspected cases in WHC, 2012-2016.

\begin{tabular}{|c|c|c|c|c|}
\hline Variables & $\mathrm{n}(\%)$ of $\mathrm{Bf}$ positives & $\mathrm{n}(\%)$ of $\mathrm{BF}$ negatives & COR $(95 \% C I)$ & AOR (95\%CI), $p$ value \\
\hline \multicolumn{5}{|r|}{ 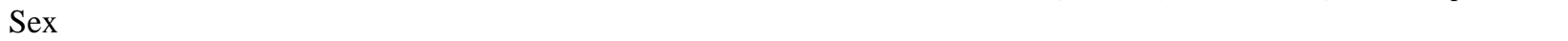 } \\
\hline Male & $232(6.4)$ & 3378 (93.6) & 1.0 & 1.0 \\
\hline Female & $202(4.5)$ & 4245 (95.5) & $1.4(1.189-1.752)$ & $1.3(1.101-1.638), 0.004$ \\
\hline \multicolumn{5}{|c|}{ Age group in years } \\
\hline$<5$ & $47(5.7)$ & 775 (94.3) & 1.0 & 1.0 \\
\hline $5-15$ & $96(6.3)$ & 963 (93.7) & $0.6(0.424-0.873)$ & $1.2(0.845-1.606), 0.352$ \\
\hline$>15$ & $291(4.7)$ & $5885(95.3)$ & $1.2(0.893-1.684)$ & $1.9(1.498-2.455), 0.000$ \\
\hline \multicolumn{5}{|l|}{ Residence } \\
\hline Urban & $141(4.7)$ & 2854 (95.3) & 1.0 & 1.0 \\
\hline Rural & $293(5.8)$ & $4769(94.2)$ & $0.8(0.653-0.987)$ & $0.8(0.660-1.003), 0.053$ \\
\hline \multicolumn{5}{|l|}{ Month } \\
\hline Sep & $33(3.7)$ & $852(96.3)$ & 1.0 & 1.0 \\
\hline Oct & $46(4.6)$ & 949 (95.4) & $0.8(0.506-1.261)$ & $0.5(0.224-1.288), 0.164$ \\
\hline Nov & $73(11.1)$ & $587(88.9)$ & $0.3(0.204-0.476)$ & $1.9(1.027-3.535), 0.041$ \\
\hline Dec & $65(8.1)$ & $733(91.9)$ & $0.4(0.284-0.671)$ & $4.2(2.374-7.560), 0.000$ \\
\hline Jan & $22(3.6)$ & $583(96.4)$ & $1.0(0.592-1.778)$ & 3.3 (1.871-5.717), 0.000 \\
\hline Feb & $27(4.6)$ & $558(95.4)$ & $0.8(0.476-1.346)$ & $1.4(0.705-2.816), 0.332$ \\
\hline Mar & $32(6.9)$ & $435(93.1)$ & $0.5(0.319-0.868)$ & $1.6(0.840-3.206), 0.174$ \\
\hline Apr & $13(2.4)$ & $539(97.6)$ & $0.3(0.134-0.494)$ & $2.0(1.122-3.543), 0.019$ \\
\hline May & $22(3.3)$ & $646(96.7)$ & $0.5(0.224-0.896)$ & $0.8(0.385-1.559), 0.474$ \\
\hline Jun & $61(7.2)$ & $785(92.8)$ & $0.2(0.107-0.360)$ & 1.5 (0.782-2.822), 0.226 \\
\hline Jul & $32(6.3)$ & $472(93.7)$ & $0.2(0.117-0.433)$ & 2.6 (1.448-4.508), 0.001 \\
\hline Aug & $8(1.6)$ & $484(98.4)$ & $0.9(0.380-2.243)$ & $2.2(1.148-4.275), 0.018$ \\
\hline \multicolumn{5}{|r|}{-2} \\
\hline 2012 & $75(5.1)$ & 1338 (94.9) & 1.0 & 1.0 \\
\hline 2013 & $73(4.4)$ & $1598(95.6)$ & $1.2(0.882-1.707)$ & $1.6(0.980-2.722), 0.059$ \\
\hline 2014 & $101(6.3)$ & 1503 (93.7) & $0.8(0.613-1.135)$ & $1.0(0.965-1.459), 0.971$ \\
\hline 2015 & $119(6.7)$ & 1647 (93.3) & $0.8(0.576-1.045)$ & $1.2(0.817-1.811), 0.335$ \\
\hline 2016 & $66(4.1)$ & $1537(95.9)$ & $1.3(0.930-1.832)$ & $1.6(1.133-2.214), 0.007$ \\
\hline
\end{tabular}

\section{DISCUSSION}

Malaria is the world's deadliest mosquito-borne disease. The African continent continues to bear the greatest burden of malaria and the greatest diversity of parasites, mosquito vectors and human victims (19). Ethiopia's complex topography and seasonal rainfall largely support its transmission, and it makes one of the public health challenges in the country.
January, April, July and August ( $p$ value <0.05) (Table 2). 
suspected and tested individuals in public health facilities of Ethiopia showed increment from about $9 \%$ in 2005 to $38 \%$ in 2015. However, the prevalence of $\mathrm{BF}$ confirmed malaria among these suspected cases over these periods ranged from $1 \%$ to $4 \%$; the highest, $(4 \%)$, was recorded in the year 2013 (11). The finding of this study is in agreement with the nationwide estimate although a minor difference was observed which might be related to the difference in sample size. Similarly, according to the Ethiopian 2011 malaria indicator survey, malaria prevalence was reported to be $1.3 \%(13,15)$ which makes our finding a bit higher than this report. However, other similar studies in Ethiopia demonstrated $14.8-25.6 \% \quad(20,21)$ prevalence which is quite higher than our finding and the national estimate. The disparity might be related to the difference in geographical location, season and the employed sample size.

The two most important species of malaria identified in this study were P. falciparum at 233 (53.7\%) and $P$. vivax at 184 (42.4\%). There were $17(3.9 \%)$ reports of mixed infections. This result was comparable with the national prevalence study where about $57 \%$ and $43 \%$ of malaria cases in Ethiopia are accounted to P. falciparum and $P$. vivax, respectively (1). Similar findings were also reported in other studies in Ethiopia $(20,22)$. Furthermore, different literatures indicated that $P$. falciparum is responsible for the most common form of malaria in Ethiopia (15). According to the WHO malaria report (7), P. falciparum is most prevalent in the African continent, and is responsible for most deaths. The report also indicated that $P$. vivax accounts for about $38 \%$ of the reported cases in Ethiopia which is concurring with the finding of this study.

In this study, the annual distribution of malaria in the five years' period showed that higher proportions of confirmed cases were documented in the months of November, December and June at $11.1 \%, 8.1 \%$ and $7.2 \%$, respectively. It was also found that patients who visited the health center in the month of December were more than 4 times likely to be malaria infected as compared with those who came in the first month of Ethiopian calendar, September, [AOR: 4.2, 95\%CI (2.374-7.560), $p$ value 0.000].
The distribution of malaria also showed a significant difference in the month of November, January, April, July and August ( $p$ value <0.05). According to different reports, in most parts of the country, the peak periods of malaria transmission occur from September to November, following the main rainy seasons, and from May to June, following the small rainy season $(12,14,15)$. This makes our report consistent with the national report. A similar finding was also reported by Yewhalaw et al. in 2013 in the country (22). The unstable malaria transmission patterns make Ethiopia prone to focal and multifocal epidemics that have on occasion caused catastrophic public health emergencies (15). The prevalence of malaria over the five years' period in the present study did not show typical trend/direction, instead there were ups and downs ranging from $4.1 \%$ to $6.3 \%$ which might indicate inconsistent intervention measures taken to reduce the burden of the disease in Ethiopia.

In this study, it was found that females were 1.3 times more likely to be positive for malaria than males [AOR: 1.3, 95\%CI (1.101-1.638), $p$ value 0.004]. This difference may be related to their relative low immune status compared with males. In this case, this finding is in line with the reports of the world malaria report (16). Similarly, it was found that patients in the age group above 15 were 1.9 times more likely to be infected with malaria than individuals under the age of 5, [AOR: 1.9 95\%CI (1.498-2.455), $p$ value 0.000]. This might be related with their frequent outdoor activities.

Due to its retrospective nature, the study lacks detailed clinical picture of patients which might have a determinant factor to show the complete picture of the study subjects. This calls for proper documentation of patient data. It would also be useful to know the percentage of malaria infected people and who were not presenting at the health center with suspected malaria. However, the report of our study will be an important source of data that will indirectly provide information concerning the implementation of malaria prevention and control measures in the study area where there is a high burden of the disease.

In conclusion, despite the many efforts that 
are in place to control it, malaria remains one of the most important causes of morbidity in our study area. The predominant identified species was $P$. falciparum. Thus, concerned stakeholders should strengthen sustainable malaria prevention and control measures to restrain the problem.

\section{ACKNOWLEDGMENTS}

The authors would like to thank laboratory staffs working at WHC who helped us during data collection.

\section{REFERENCES}

1. World Health Organization. World Malaria Report 2005. 20 Avenue Appia, 1211 Geneva 27, Switzerland. Available at: <http://apps.who.int/iris/bitstream/10665/43213/1/ 9241593199_eng.pdf>. Accessed on 27 Dec 2016.

2. Rowe K, Rowe Y, Snow W, et al. The burden of malaria mortality among African children in the year 2000. Int Journal of Epidemiol 2006; 35:691704.

3. Ghebreyesus I, Deresa W, Written KH, et al. The epidemiology and Ecology of health and death in Ethiopia. Addis Ababa Ethiopia J-Health Deve 2007; 21(2).

4. Grant A, Roussilhon C, Paul R, Sakuntabhai A. The genetic control of immunity to Plasmodium infection. BMC Immunology 2015; 16:14.

5. World Health Organization. Eliminating malaria, WHO/HTM/GMP/2016.3; 20 Avenue Appia, 1211 Geneva 27, Switzerland. Available at: <http://www.who.int/about/licensing/copyright_for $\mathrm{m} / \mathrm{en} /$ index.html $>$. Accessed on 1 Jan 2017.

6. World Health Organization. Global technical strategy for malaria 2016-2030; 20 Avenue Appia, 1211 Geneva 27, Switzerland. Available at: < www.who.int/about/licensing/ copyright_form/en/index.html>. Accessed on 1 Jan 2017.

7. World Health Organization; WHO global malaria program, World malaria report 2014. Available at <www.who.int/malaria>. Accessed on $15 \mathrm{Dec}$ 2016.

8. Lengerar C. Insecticide treated nets for malaria control real gains. Bulletin of WHO, Geneva Switzerland, World Health Organization 2004; 8284.

9. Epidemiology and distribution of malaria in Ethiopia. Avalable at: <http://moodle.digital- campus.org/mod/page/view.php?id=14803>. Acsessed on 1 Jan 2017.

10. FDRE, MOH. Malaria prevention and control programs. Available at: <http://www.moh.gov.et/malaria?p_p_auth=Ws9d 2X5o\&p_p_id=77\&p_p_lifecycle=0\&p_p_state $=$ maximized\&p_p_mode=view\&_77_struts_action $=\% 2$ Fjournal_content_search\%2Fsearch $>$. Accessed on; 4 Jan 2017

11. World Health Organization. World Malaria Report 2016. Regional Profile. Available at: <http://www.who.int/malaria/publications/worldmalaria-report-2016/WMR-2016-regionalprofiles.pdf?ua=1>. Accessed on 4 Jan 2017.

12. FDRE, MoH. Malaria Epidemiological profile. Available at: <http://www.moh.gov.et/malaria>. Accessed; on 4 Jan 2017.

13. President's malaria initiative. Ethiopia-malaria operational plan FY 2016.

14. African Union update on malaria control in Africa. Special summit of Africa Union on HIV/AIDs, TB and malaria (ATM): Abuja, Nigeria 2006.

15. The Ethiopian Health and Nutrition Research Institute \& partners. Ethiopia National Malaria Indicator Survey; Addis Ababa Ethiopia 2011.

16. WHO Global malaria programme. World Malaria Report 2014; 20 Avenue Appia, 1211 Geneva 27, Switzerland.

17. Cheesbrough M. District laboratory practice in tropical countries. Part one, Second Edition. Cambridge University 2009; p244-245.

18. Tangpukdee N, Duangdee C, Wilairatana $\mathrm{P}$, Krudsood S. Malaria Diagnosis: A Brief Review. Korean J Parasitol 2009; 47(2): 93.

19. Ghansah A, Amenga-Etego L, Amambua-Ngwa A, et al. Monitoring parasite diversity for malaria elimination in sub-Saharan Africa. Science. 2014; 345(6202):1297-8.

20. Yihenew G, Adamu H, Petros B. The impact of cooperative social organization on reducing the prevalence of malaria and intestinal parasite infections in Awramba, a rural community in South Gondar, Ethiopia. Interdiscip Perspect Infect Dis 2014:378780.

21. Deressa W, Ali A, Enqusellassie F. "Self-treatment of malaria in rural communities, Butajira, southern Ethiopia," Bulletin of the World Health Organization, 2003; 81(4): 261.

22. Yewhalaw D Getachew Y, Tushune K, et al. The effect of dams and seasons on malaria incidence and anopheles abundance in Ethiopia. BMC Infect Di 2013; 13: 161.

DOI: http://dx.doi.org/10.4314/ejhs.v27i5.4 